\title{
Feshbach resonances and weakly bound molecular states of boson-boson and boson-fermion NaK pairs
}

\author{
Alexandra Viel $^{*}$ and Andrea Simoni ${ }^{\dagger}$ \\ Institut de Physique de Rennes, UMR 6251, CNRS \& Université de Rennes 1, F-35042 Rennes, France
}

(Received 28 January 2016; published 5 April 2016)

\begin{abstract}
We conduct a theoretical study of magnetically induced Feshbach resonances and near-threshold bound states in isotopic $\mathrm{NaK}$ pairs. Our calculations accurately reproduce Feshbach spectroscopy data on $\mathrm{Na}^{40} \mathrm{~K}$ and explain the origin of the observed multiplets in the $p$ wave [Phys. Rev. A 85, 051602(R) (2012)]. We apply the model to predict scattering and bound state threshold properties of the boson-boson $\mathrm{Na}^{39} \mathrm{~K}$ and $\mathrm{Na}^{41} \mathrm{~K}$ systems. We find that the $\mathrm{Na}^{39} \mathrm{~K}$ isotopic pair presents broad magnetic Feshbach resonances and favorable ground-state features for producing nonreactive polar molecules by two-photon association. Broad $s$-wave resonances are also predicted for $\mathrm{Na}^{41} \mathrm{~K}$ collisions.
\end{abstract}

DOI: 10.1103/PhysRevA.93.042701

\section{INTRODUCTION}

Ultracold gases are extraordinary systems to investigate fundamental quantum phenomena in a highly controllable environment, leading to a wealth of spectacular experimental and theoretical results. More than a decade ago, the experimental production of ultracold mixtures of alkali-metal gases added a new twist to the cold-atom field, paving the way toward the study of few- and many-body phenomena absent in a pure homonuclear gas. Some examples include recent experiments with polaronic impurities [1-3], the formation of chemically reactive [4] or nonreactive [5] ultracold polar molecules, and theoretical studies of phase diagrams [6] and pairing in imbalanced Fermi systems [7,8].

In this context, magnetic Feshbach resonances (FRs) proved to be a powerful and versatile tool to widely tune few-body interactions [9], allowing one to explore in a controlled way regimes from the noninteracting ideal behavior to strongly interacting systems. A FR also offers the possibility to associate pairs of atoms in weakly bound molecular states using time-dependent magnetic fields [10]. Such molecules have an intrinsic interest due to their long-range nature. Moreover, depending on their spin and spatial structure, they can be used as a convenient initial state for producing polar molecules in the ground state via stimulated Raman processes [4,5].

Applications based on resonances require an accurate characterization of the scattering dynamics and of the properties of bound states near the dissociation threshold. Fortunately, theory can predict from a small amount of experimental data the location and the width of magnetic resonances and the relevant molecular state properties. In fact, to date highly quantitative models exist for most alkali-metal isotopic pairs [9]. In this work, we focus on $\mathrm{NaK}$ mixtures, a system composed of two species that can be individually cooled to ultracold temperatures.

Experiments with $\mathrm{Na}$ and the fermionic isotope ${ }^{40} \mathrm{~K}$ are currently being performed at MIT, where heteronuclear FR spectra have been discovered and interpreted based on simplified asymptotic models [11]. Magnetic association [12]

\footnotetext{
*alexandra.viel@univ-rennes1.fr

†andrea.simoni@univ-rennes1.fr
}

and, more recently, transfer to the ro-vibrational ground state of the dimer have also been demonstrated [13]. Accurate BornOppenheimer potentials for the ground and excited states have been built and used to study the adiabatic transfer of a Feshbach molecule to the ground rovibrational state [14]. However, a comprehensive account of scattering and bound-state features for this boson-fermion mixture in the electronic ground state is still lacking. In addition, near-threshold properties for the boson-boson pairs $\mathrm{Na}^{39} \mathrm{~K}$ and $\mathrm{Na}^{41} \mathrm{~K}$ are still unknown. The aim of this work, therefore, is on the one hand to provide a more complete picture of the Feshbach physics of $\mathrm{Na}^{40} \mathrm{~K}$, and on the other hand to provide theoretical predictions for the two purely bosonic pairs, for which experiments are underway in a few groups worldwide. We study both scattering and bound states for an extensive set of hyperfine states, and we discuss the experimental implications of our results for interaction control and molecule production.

The paper is organized as follows. Section II introduces our theoretical approach and the Born-Oppenheimer potential optimization procedure based on known experimental data. Section III presents results and discussions for the boson-fermion and the boson-boson pairs. Some experimental implications of our results are discussed. A conclusion in Sec. IV ends this work.

\section{METHODS}

\section{A. Computational approach}

We solve the time-independent Schrödinger equation for bound and scattering states in the well-known framework of the close-coupling approach to molecular dynamics. Briefly, in our approach a basis of Hund's case (b) molecular states is used to expand the total wave function at each value of the interatomic distance $R$. In Hund's case (b) the spin state of the dimer is represented as $\left|S M_{S} I M_{I}\right\rangle$, with $\vec{S}$ and $\vec{I}$ the electronic and nuclear spin angular momentum, respectively [15]. The description of the diatomic is completed by assigning the $\ell$ and $m$ quantum numbers relative to the orbital angular momentum $\vec{\ell}$ of the atoms about their center of mass. In this basis, the electrostatic Born-Oppenheimer potentials are represented by diagonal matrices with entries the singlet $X^{1} \Sigma^{+}$and triplet 
$a^{3} \Sigma^{+}$molecular symmetry adiabatic potential energy curves (see below).

The other interactions relevant for the ultracold regime included in this work are the atomic hyperfine interaction $H_{\mathrm{hf}}=\sum_{k=a, b} A_{k} \vec{s}_{k} \cdot \vec{\imath}_{k}$, the anisotropic spin-spin coupling $H_{\mathrm{ss}}=\alpha^{2} R^{-3}\left[\vec{s}_{a} \cdot \vec{s}_{b}-3\left(\vec{s}_{a} \cdot \hat{R}\right)\left(\vec{s}_{b} \cdot \hat{R}\right)\right]$, and the Zeeman interaction energy with the external magnetic field $H_{\mathrm{Z}}=$ $\mu_{B} \sum_{k=a, b}\left(g_{s} \vec{s}_{k}+g_{i} \vec{\imath}_{k}\right) \cdot \vec{B}$. Here $\vec{s}_{k}$ and $\vec{\imath}_{k}$ are the electron and nuclear spin of the individual atoms, $A_{k}$ is the respective ground-state atomic hyperfine constant for atoms $a$ and $b, g_{s}$ and $g_{i}$ are the electron and nuclear gyromagnetic ratios, and $\alpha$ and $\mu_{B}$ are the fine-structure constant and the Bohr magneton, respectively. Such atomic interactions can be expressed in the molecular Hund's case (b) computational basis using standard methods of angular momentum algebra (see, e.g., [16]).

Bound-state calculations are performed using a variable grid approach allowing one to represent over a sufficiently small number of points the rapid oscillations at short range and the long-range tail of the dimer wave function [17]. For scattering calculations, we use the variable-step Gordon propagation or the renormalized Numerov algorithm to efficiently solve the coupled-channel Schrödinger equation [18]. Once the solution has been built in the computational basis, a frame transformation is applied to express the solution in an asymptotically diagonal representation before using a standard matching procedure to extract the reactance matrix $K$ [18].

\section{B. Optimization of the molecular potential}

We adopt for this work the $X^{1} \Sigma^{+}$and the $a^{3} \Sigma^{+}$electronic ground-state potential of the $\mathrm{NaK}$ molecule proposed in Ref. [19]. A minor modification is made to ensure a continuous and continuously differentiable expression by fine-tuning the parameters given in [19]. First, starting from the asymptotic long-range $U_{\mathrm{LR}}(R)$ expressions, we numerically enforce continuity at the switching points $R_{i}$ and $R_{0}$. In addition, smooth damping functions are preferred to the published abrupt change of the potential between the short-range repulsive part, the inner well, and the long-range part. The resulting continuously differentiable expression is given by

$$
\begin{aligned}
V(R)= & U_{\mathrm{SR}}(R)\left\{1-\tanh \left[\beta\left(R-R_{i}\right)\right]\right\} \\
& \times\left\{1-\tanh \left[\beta\left(R-R_{0}\right)\right]\right\} / 4 \\
& +U(R)\left\{1+\tanh \left[\beta\left(R-R_{i}\right)\right]\right\} \\
& \times\left\{1-\tanh \left[\beta\left(R-R_{0}\right)\right]\right\} / 4 \\
& +U_{\mathrm{LR}}(R)\left\{1+\tanh \left[\beta\left(R-R_{i}\right)\right]\right\} \\
& \times\left\{1+\tanh \left[\beta\left(R-R_{0}\right)\right]\right\} / 4,
\end{aligned}
$$

where the parametrized functions $U_{\mathrm{SR}}(R), U(R)$, and $U_{\mathrm{LR}}(R)$ and the switching points $R_{i}$ and $R_{0}$ are taken from the work of Tiemann and co-workers [19]. Note that there is a typo in Table I of Ref. [19], i.e., $10^{6}$ should be replaced by $10^{8}$ for the $B$ constant value. A value of $80 a_{0}^{-1}$ has been found to be suitable for the control parameter $\beta$ of the damping function for the two electronic states. With an infinite $\beta$ value, the original potential curves are recovered.

We are now in the position to perform close-coupling calculations for different initial channels. We will conven-
TABLE I. Singlet and triplet scattering lengths $a_{\mathrm{S}, \mathrm{T}}$ obtained according to our optimized potentials for different $\mathrm{NaK}$ isotopic pairs.

\begin{tabular}{lcr}
\hline \hline Isotope & $a_{\mathrm{S}}\left(a_{0}\right)$ & $a_{\mathrm{T}}\left(a_{0}\right)$ \\
\hline $\mathrm{Na}^{39} \mathrm{~K}$ & 255 & -84 \\
$\mathrm{Na}^{40} \mathrm{~K}$ & 63 & -838 \\
$\mathrm{Na}^{41} \mathrm{~K}$ & -3.65 & 267 \\
\hline \hline
\end{tabular}

tionally label each asymptotic channel by specifying the separated-atom NaK state $\left|f_{a}, m_{f_{a}}\right\rangle+\left|f_{b}, m_{f_{b}}\right\rangle$ with $\vec{f}_{k}=$ $\vec{s}_{k}+\vec{\imath}_{k}(k=a, b)$ to which the latter adiabatically correlate as $B$ tends to zero. FRs have been observed experimentally in the collision between $\mathrm{Na}$ in $|1,1\rangle$ and ${ }^{40} \mathrm{~K}$ in $\left|9 / 2, m_{f}\right\rangle$, with $m_{f_{b}}=-9 / 2,-7 / 2,-5 / 2$, and $-3 / 2$ [11]. We compute the $s$-wave scattering length $a$ in the relevant channels and search for resonances as poles of $a$ as a function of magnetic field. We also search for $p$-wave resonances by locating maxima in the partial $p$-wave elastic cross sections at a fixed collision energy of $1 \mu \mathrm{K}$. The observed resonance locations are not reproduced accurately by the original potentials. However, a simple modification consisting in introducing the correction terms

$$
V_{\text {corr }}^{(i)}(R)=s^{(i)} / \cosh \left[\frac{R-R_{e}^{(i)}}{R_{0}^{(i)}}\right]^{2} \text { for } i=1,3
$$

near the bottom of the $X^{1} \Sigma^{+}$and $a^{3} \Sigma^{+}$electronic potentials enables us to model the experimentally measured spectra. More specifically, using $R_{e}^{(1)}=6.61 a_{0}, R_{e}^{(3)}=10.29 a_{0}$, and $R_{0}^{(1,3)}=1.5 a_{0}$, a Levenberg-Marquardt algorithm is applied to determine two optimal $s^{(i)}$ parameters,

$$
\begin{aligned}
& s^{(1)}=-0.37247 \times 10^{-4} E_{h}, \\
& s^{(3)}=-0.16385 \times 10^{-5} E_{h} .
\end{aligned}
$$

In the fitting procedure, we included three $s$-wave resonances at $78.3,88.2$, and $81.6 \mathrm{G}$ and an average position of the $p$-wave resonances appearing as a multiplet around $19.19 \mathrm{G}$ [11]. For the $p$-wave calculation, we initially neglect the spin-coupling term responsible for the multiplet structure, thus avoiding possible incorrect labeling of the closely spaced $p$-wave resonances.

After optimization, the resonance positions are theoretically reproduced with a reduced $\chi^{2}=0.57$. On a more physical basis, the artificial control parameters are usually translated in corresponding singlet and triplet scattering lengths $a_{\mathrm{S}, \mathrm{T}}$ (see Table I). Our optimized boson-fermion model can also be used for predicting the properties of boson-boson isotopes. Within the Born-Oppenheimer approximation, which is expected to be accurate for all but the lightest species [20], it is sufficient to change the value of the reduced mass in the Hamiltonian to compute $a_{\mathrm{S}, \mathrm{T}}$. Note, however, that if the number of bound states in our nominal potentials turn out to be incorrect, the predicted $a_{\mathrm{S}, \mathrm{T}}$ (and hence the results of the coupled model) will be systematically shifted.

We begin our analysis with a detailed discussion of the resonances for the $\mathrm{Na}^{40} \mathrm{~K}$ boson-fermion mixture in Sec. III A. 
TABLE II. List of the $s$-wave FR for $\mathrm{Na}^{40} \mathrm{~K}$. Our theoretical resonance positions $B_{\text {res }}$ are compared with the experimental data $B_{\text {exp }}$ from Ref. [11]. The locations where the scattering length vanishes, $B_{\mathrm{ZC}}$, the effective range on resonance, $r_{\mathrm{eff}}^{\text {res }}$, the resonance strength parameter, $s_{\text {res }}$, and the fitting parameters, $a_{\mathrm{bg}}, \varepsilon$, and $\Delta$, defined in Eq. (4) are reported in columns 4-9. See the text for more details.

\begin{tabular}{|c|c|c|c|c|c|c|c|c|}
\hline $\mathrm{Na}^{40} \mathrm{~K}$ channel & $B_{\exp }(\mathrm{G})$ & $B_{\text {res }}(\mathrm{G})$ & $B_{\mathrm{ZC}}(\mathrm{G})$ & $r_{\mathrm{eff}}^{\mathrm{res}}\left(a_{0}\right)$ & $s_{\text {res }}$ & $a_{\mathrm{bg}}\left(a_{0}\right)$ & $\varepsilon\left(\mathrm{G}^{-1}\right)$ & $\Delta(\mathrm{G})$ \\
\hline$|1,1\rangle+|9 / 2,-9 / 2\rangle$ & $\begin{array}{l}78.3 \\
88.2\end{array}$ & $\begin{array}{l}77.78 \\
88.68\end{array}$ & $\begin{array}{l}72.23 \\
79.82\end{array}$ & $\begin{array}{l}-0.668 \\
137.0\end{array}$ & $\begin{array}{l}0.682 \\
9.92\end{array}$ & -619.3 & $-1.2 \times 10^{-3}$ & $\begin{array}{l}-5.5 \\
-8.8\end{array}$ \\
\hline$|1,1\rangle+|9 / 2,-7 / 2\rangle$ & $\begin{array}{r}81.6 \\
89.8 \\
108.6\end{array}$ & $\begin{array}{r}81.42 \\
89.82 \\
108.91\end{array}$ & $\begin{array}{l}81.18 \\
83.61 \\
96.86\end{array}$ & $\begin{array}{l}-8.39 \times 10^{4} \\
-48.4 \\
141.0\end{array}$ & $\begin{array}{l}0.0120 \\
0.517 \\
12.3\end{array}$ & -552.7 & $-1.1 \times 10^{-3}$ & $\begin{array}{l}-0.23 \\
-6.2 \\
-16.0\end{array}$ \\
\hline$|1,1\rangle+|9 / 2,-5 / 2\rangle$ & $\begin{array}{l}96.5 \\
106.9 \\
138\end{array}$ & $\begin{array}{r}96.39 \\
106.54 \\
136.82\end{array}$ & $\begin{array}{r}95.75 \\
98.83 \\
110.53\end{array}$ & $\begin{array}{l}-4.84 \times 10^{4} \\
-90.9 \\
142.0\end{array}$ & $\begin{array}{l}0.0205 \\
0.426 \\
14.58\end{array}$ & -496.4 & $-1.0 \times 10^{-3}$ & $\begin{array}{r}-0.6 \\
-7.5 \\
-26.2\end{array}$ \\
\hline$|1,1\rangle+|9 / 2,-3 / 2\rangle$ & $\begin{array}{l}116.9 \\
129.5 \\
175\end{array}$ & $\begin{array}{l}117.19 \\
130.36 \\
177.44\end{array}$ & $\begin{array}{l}115.62 \\
119.85 \\
135.35\end{array}$ & $\begin{array}{l}-3.46 \times 10^{4} \\
-120.0 \\
143.0\end{array}$ & $\begin{array}{l}0.0283 \\
0.379 \\
17.3\end{array}$ & -443.1 & $-8.5 \times 10^{-4}$ & $\begin{array}{r}-1.2 \\
-9.8 \\
-41.7\end{array}$ \\
\hline
\end{tabular}

\section{RESULTS}

\section{A. $\mathrm{Na}^{40} \mathrm{~K}$}

We now perform extensive close-coupling calculations with the optimized potentials described in Sec. II B. Table II summarizes our findings for the $s$-wave for magnetic fields up to $1000 \mathrm{G}$ for different hyperfine levels. We report in the table the positions of the poles $B_{\text {res }}$ observed in the calculated $s$-wave scattering length $a$ as well as the nearby zero-crossing field $B_{\mathrm{ZC}}$ where $a$ vanishes. The experimental data of Ref. [11] are also reproduced in the table.

The good quality of the theoretical model after optimization is confirmed by the very good agreement (below $0.5 \mathrm{G}$ ) illustrated in the table for all narrow features experimentally observed in different hyperfine combinations. A larger discrepancy of $\sim 1 \mathrm{G}$ is found on broader resonances, which may, however, be more difficult to locate experimentally with accuracy. No additional $s$-wave features are found with respect to the experiment.

To extract the magnetic width $\Delta$, the scattering length $a(B)$ obtained for each incoming channel is fitted according to a formula appropriate for overlapping resonances [21],

$$
a(B)=a_{\mathrm{bg}}(1+\varepsilon B) \prod_{i}^{N}\left(1-\frac{\Delta_{i}}{B-B_{\mathrm{res}, i}}\right),
$$

in which a linear variation of $a_{\mathrm{bg}}$ as a function of the magnetic field is assumed. Equation (4) reduces to the wellknown standard expression $a(B)=a_{\mathrm{bg}}\left[1-\Delta /\left(B-B_{\text {res }}\right)\right]$ if resonances are isolated and the background scattering length is locally constant. The fitting of the scattering length with Eq. (4) has been carried out for magnetic fields spanning a $\pm 4 \Delta$ region around each resonance. For overlapping cases, the largest $\Delta$ was taken to define the fitting interval. We only report in the table the corresponding $\Delta_{i}, a_{\mathrm{bg}}$, and $\varepsilon$ parameters for which Eq. (4) reproduces the numerical data to an accuracy better than $5 \%$ in either the relative error or in the absolute error measured in units of the van der Waals radius,

$$
R_{\mathrm{vdW}}=\frac{1}{2}\left(\frac{2 \mu C_{6}}{\hbar^{2}}\right)^{1 / 4}
$$

The latter quantity ( $\approx 53 a_{0}$ for $\mathrm{NaK}$ ) represents the natural value of $a$ for scattering in a van der Waals potential [9].

In addition, for each resonance we extract the effective range $r_{\text {eff }}$ defined through the low-energy expansion of the elastic reactance matrix element,

$$
\frac{k}{K(E, B)}=-\frac{1}{a(B)}+\frac{1}{2} r_{\mathrm{eff}}(B) k^{2},
$$

computed at the resonance value $r_{\mathrm{eff}}^{\mathrm{res}} \equiv r_{\mathrm{eff}}\left(B_{\mathrm{res}}\right)$ by a linear fit of $k / K\left(E, B_{\text {res }}\right)$ as a function of collision energy $E=$ $\hbar^{2} k^{2} /(2 \mu)$ in an appropriate energy domain. We introduce a corresponding intrinsic resonance length $R^{*}$ to characterize the resonance strength defined as

$$
r_{\mathrm{eff}}=-2 R^{*}+\frac{2}{3 \pi} \Gamma^{2}\left(\frac{1}{4}\right) R_{\mathrm{vdW}} .
$$

In the case of isolated resonances, the resonance length can be expressed in terms of scattering background and resonance parameters and of the magnetic moment difference $\delta \mu$ between the open and the closed channel as $R^{*}=\hbar^{2} /\left(2 \mu a_{\mathrm{bg}} \delta \mu \Delta\right)$ [9]. As remarked in the Supplemental Material of Ref. [22], Eq. (7) also holds for overlapping resonances to the extent that they are not directly interacting. According to the relative value of the length $R^{*}$ being much larger (much smaller) than the van der Waals length, resonances are classified as being open channel (closed channel) dominated. The resonance strength parameter defined as

$$
S_{\mathrm{res}}=\frac{0.956 R_{\mathrm{vdW}}}{R^{*}}
$$

and listed in Table II is therefore a useful dimensionless indicator of the resonance character [9].

It is interesting to compare the present close-coupling data with the results of an asymptotic bound-state model used for interpretation in Ref. [11] (not shown in the table). Considering the simplicity of the latter, the agreement is good as far as the resonance position and the width of the largest features is concerned. The most serious discrepancy concerns the width of the narrow $s$-wave resonances, which are underestimated by more than one order of magnitude by the asymptotic model. 
Feshbach molecules formed by magnetic association can be a good starting point to form molecules in the absolute rovibrational ground state using two-photon transfer schemes. A first constraint to be taken into account to achieve such a transfer is that according to electric dipole selection rules, only Feshbach molecular states of significant singlet character can be coupled to ground-state singlet molecules if singlet excited states are used as a bridge. If the initial Feshbach molecule turns out to have mostly triplet character, one can use excited electronic states of mixed singlet-triplet character as a bridge, an approach suggested for $\mathrm{NaK}$ in [14] and successfully adopted recently to form $\mathrm{Na}^{40} \mathrm{~K}$ in the absolute ground state by a STIRAP two-photon process $[13,23]$. Moreover, the radial overlap between the excited intermediate state and both the initial and the target ground-state molecule must be significant. To gain more insight into the resonance nature and to get a hint at the expected efficiency of two-photon processes, we perform bound-state coupled-channel calculations. A detailed analysis having already been performed in [14] for $\mathrm{Na}^{40} \mathrm{~K}$, here we just stress the main elements for the sake of comparison with the following analysis of the boson-boson pairs.

We depict in Fig. 1, for example, the scattering length and the evolution of the molecular levels near the broadest resonance in the hyperfine absolute ground state, which has been successfully used as starting point for STIRAP association [13]. The corresponding average electronic spin $\left\langle\vec{S}^{2}\right\rangle$ is also shown as a function of internuclear distance by a color code in the lower panel. The nearly pure triplet character of this molecular state is in principle unfavorable for the production of ground-state singlet molecules through the excited singlet manifold. Inspection of the lower panel of Fig. 1 might suggest working closer to resonance to increase the single character as the molecular state mixes with the scattering continuum. However, as already noted in Ref. [14], such admixture comes at the price of a delocalization of the wave function at larger distances, and thus a decreased overlap with
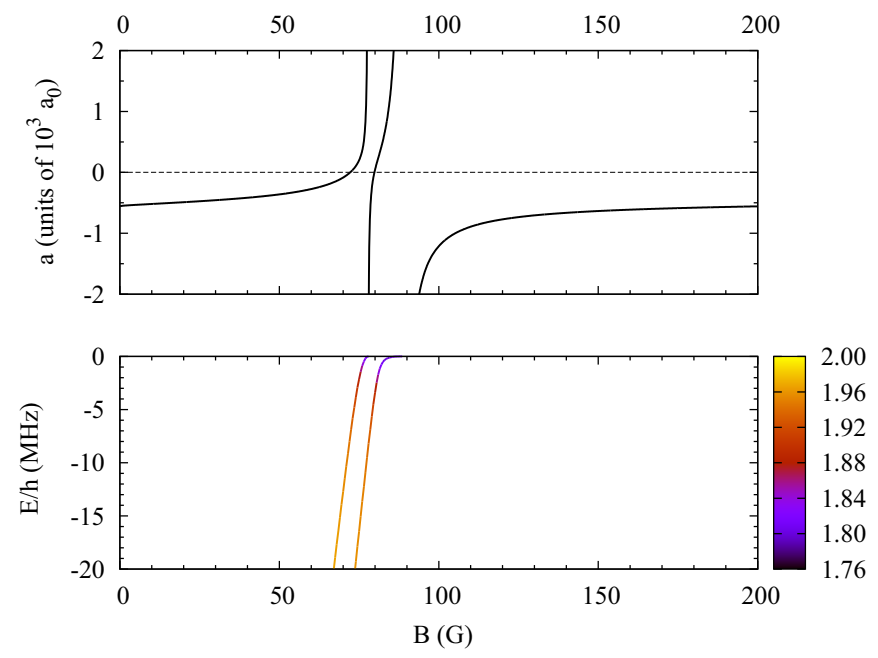

FIG. 1. Scattering length $a$ as a function of the magnetic field $B$ for $\mathrm{Na}|1,1\rangle+{ }^{40} \mathrm{~K}|9 / 2,-9 / 2\rangle s$-wave collisions (top panel). Corresponding molecular energy levels as a function of $B$ are shown in the lower panel. The density code denotes the average spin $\left\langle\vec{S}^{2}\right\rangle$ of the molecule.
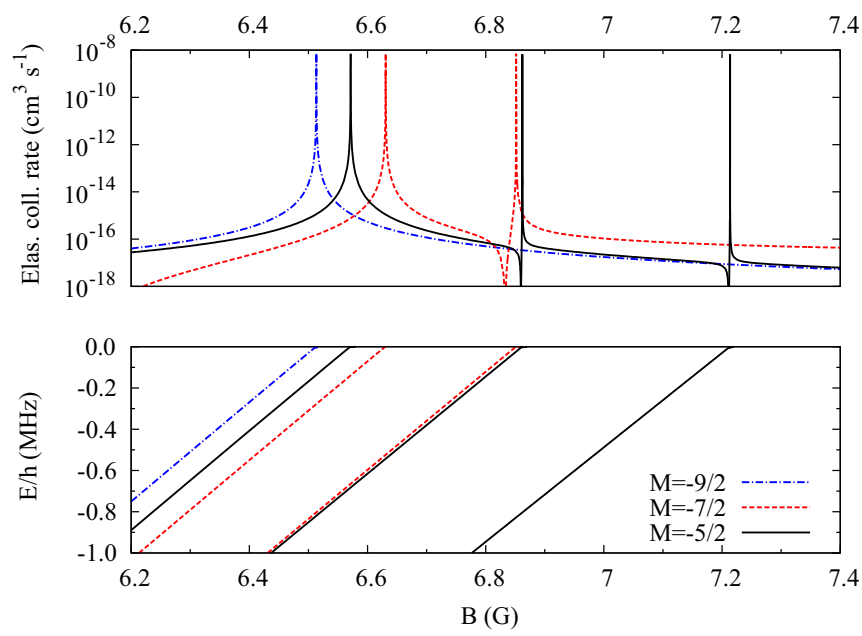

FIG. 2. Elastic rate computed at a collision energy of $1 \mu \mathrm{K}$ as a function of the magnetic field $B$ for $\mathrm{Na}|1,1\rangle+{ }^{40} \mathrm{~K}|9 / 2,-9 / 2\rangle$ $p$-wave collisions (top panel). Corresponding molecular energy levels as a function of $B$ are shown in the lower panel. Curves are resolved according to the projection $M$ of total angular momentum.

the intermediate excited state. Such strong triplet character is a common feature of the molecular states associated with all of the broadest resonances in Table II, which is in fact to a good approximation a common molecular state in the triplet potential with a different projection $m_{f}$ of the total hyperfine angular momentum $\vec{f}=\vec{S}+\vec{I}$. In conclusion, use of bridge spin-orbit coupled states to help enhance the transfer efficiency seems necessary for the boson-fermion pair [14]. We will show below that the situation is significantly different for the boson-boson mixtures.

In addition to the observed $s$ - and $p$-wave features, additional $p$-wave resonances are also predicted by our model. We compute the elastic collision rate up to $B=1000 \mathrm{G}$ and present a restricted magnetic-field range in the upper panel of Fig. 2. The lower panel depicts the energy levels of the molecular states responsible for each resonance. Resonance features are detected by local maxima in the elastic collision rate as well as in the inelastic probabilities. The positions of these maxima agree to better than $0.02 \mathrm{G}$ for all except the two features around $21.9 \mathrm{G}$ in the $|1,1\rangle+|9 / 2,-7 / 2\rangle$ channel, for which the differences are $0.05 \mathrm{G}$. It turns out to be easier to extract the location of the resonances from the inelastic probabilities. These positions are summarized in Table III together with the position of the local maxima in the elastic collision rate when no inelastic processes are present.

The $p$-wave multiplets observed in Ref. [11] and reproduced in Table III are surprising at first glance since the spin-spin interaction typically gives rise to doublets [24]. The nature and multiplicity of such a magnetic spectrum can be rationalized starting from a picture in which the spin interaction is at first neglected. In this situation, the total internal spin projection $m_{f}=m_{f_{a}}+m_{f_{b}}$ is an exactly conserved quantum number. Let us consider for definiteness the case of two free atoms with $m_{f}=-7 / 2$. Moreover, let us restrict ourselves to $\ell=1$, since the $\ell>1$ contributions are vanishingly small at the present very low collision energies due to centrifugal repulsion. The projection $M$ of 
TABLE III. Theoretical magnetic-field locations $B_{\text {res }}$ of $p$-wave FR for $\mathrm{Na}^{40} \mathrm{~K}$ for projection of total angular momentum $M$ in different hyperfine atomic channels. Calculations are performed at a collision energy of $1 \mu \mathrm{K}$. Available experimental values $B_{\text {exp }}$ are also reported [11].

\begin{tabular}{|c|c|c|c|}
\hline $\mathrm{Na}^{40} \mathrm{~K}$ channel & $M$ & $B_{\text {res }}(\mathrm{G})$ & $B_{\exp }(\mathrm{G})$ \\
\hline$|1,1\rangle+|9 / 2,-9 / 2\rangle$ & $\begin{array}{l}-9 / 2 \\
-5 / 2 \\
-7 / 2 \\
-7 / 2 \\
-5 / 2 \\
-5 / 2 \\
-7 / 2 \\
-9 / 2 \\
-5 / 2 \\
-9 / 2 \\
-7 / 2 \\
-5 / 2 \\
-7 / 2 \\
-5 / 2\end{array}$ & $\begin{array}{r}6.51 \\
6.57 \\
6.63 \\
6.85 \\
6.86 \\
7.21 \\
18.01 \\
18.36 \\
19.32 \\
19.39 \\
19.47 \\
20.72 \\
20.82 \\
22.43\end{array}$ & $\begin{array}{l} \\
19.12 \\
19.18 \\
19.27\end{array}$ \\
\hline$|1,1\rangle+|9 / 2,-7 / 2\rangle$ & $\begin{array}{l}-5 / 2 \\
-7 / 2 \\
-7 / 2 \\
-5 / 2 \\
-3 / 2 \\
-3 / 2 \\
-5 / 2 \\
-3 / 2 \\
-7 / 2 \\
-5 / 2 \\
-7 / 2 \\
-5 / 2 \\
-3 / 2 \\
-7 / 2 \\
-3 / 2 \\
-5 / 2 \\
-3 / 2\end{array}$ & $\begin{array}{r}7.55 \\
7.62 \\
7.88 \\
7.90 \\
7.90 \\
8.27 \\
8.33 \\
8.87 \\
20.09 \\
21.76 \\
21.91 \\
23.49 \\
23.53 \\
23.60 \\
25.33 \\
25.61 \\
28.01\end{array}$ & 23.19 \\
\hline$|1,1\rangle+|9 / 2,-5 / 2\rangle$ & $\begin{array}{l}-5 / 2 \\
-5 / 2 \\
-3 / 2 \\
-3 / 2 \\
-5 / 2 \\
-1 / 2 \\
-1 / 2 \\
-3 / 2 \\
-1 / 2 \\
-5 / 2 \\
-5 / 2 \\
-3 / 2 \\
-3 / 2 \\
-1 / 2 \\
-5 / 2 \\
-1 / 2 \\
-3 / 2 \\
-1 / 2\end{array}$ & $\begin{array}{r}8.87 \\
9.34 \\
9.33 \\
9.82 \\
9.88 \\
9.89 \\
10.46 \\
10.61 \\
11.50 \\
24.80 \\
27.20 \\
27.28 \\
29.61 \\
29.88 \\
29.93 \\
32.57 \\
33.04 \\
36.97\end{array}$ & $\begin{array}{c}9.23 \\
9.60 \\
\\
\\
\\
\\
29.19 \\
29.45 \\
29.52\end{array}$ \\
\hline$|1,1\rangle+|9 / 2,-3 / 2\rangle$ & $\begin{array}{r}-3 / 2 \\
-1 / 2 \\
-3 / 2 \\
-1 / 2 \\
-3 / 2 \\
1 / 2\end{array}$ & $\begin{array}{l}11.35 \\
11.95 \\
12.12 \\
13.04 \\
13.20 \\
13.20\end{array}$ & $\begin{array}{l}12.51 \\
12.68\end{array}$ \\
\hline
\end{tabular}

TABLE III. (Continued.)

\begin{tabular}{rccc}
\hline \hline $\mathrm{Na}^{40} \mathrm{~K}$ channel & \multicolumn{1}{c}{$B_{\text {res }}(\mathrm{G})$} & $B_{\exp }(\mathrm{G})$ \\
\hline $1 / 2$ & 14.30 & \\
$-1 / 2$ & 14.55 & \\
$1 / 2$ & 16.31 & \\
$-3 / 2$ & 32.00 & \\
$-3 / 2$ & 35.74 & \\
$-1 / 2$ & 36.10 & \\
$-1 / 2$ & 39.87 & 39.39 \\
$1 / 2$ & 40.37 & 39.86 \\
$-3 / 2$ & 40.42 & \\
$1 / 2$ & 45.18 & \\
$-1 / 2$ & 45.85 & \\
$1 / 2$ & 53.21 & \\
\hline
\end{tabular}

the total angular momentum, which is strictly conserved, can then only take values $-9 / 2,-7 / 2$, and $-5 / 2$. Within this restricted model and fixing $f=7 / 2$, one can build six molecular states with projections $\left\{m_{f} m\right\}=\{-7 / 2 ; 0, \pm 1\},\{-5 / 2$; $0,-1\},\{-3 / 2 ;-1\}$ that are degenerate, since both $\vec{f}$ and $\vec{\ell}$ are strictly conserved in the absence of anisotropic spin-spin and of the Zeeman interaction [16].

If $B=0$ and the spin-spin interaction does not vanish, conservation of total angular momentum $\vec{F}=\vec{f}+\vec{\ell}$ guarantees that the six molecular states will give rise to one triply degenerate level with $F=9 / 2$ corresponding to $M=-9 / 2,-7 / 2,-5 / 2$, one doubly degenerate level with $F=7 / 2$ and with $M=-7 / 2,-5 / 2$, and one singly degenerate level with $F=5 / 2$ and with $M=-5 / 2$. The energy differences between the three groups is small due to the weakness of the spin-spin interaction. However, the mixing of the different $\left\{m_{f}, m\right\}$ within each block of given $M$ can be strong. In fact, as shown in Fig. 3, even in zero field the values of $m_{f}$, and hence of $m$, are undefined with the exception of the bound level with $M=-9 / 2$, which being essentially isolated retains to high accuracy its $m_{f}=-7 / 2$ and $m=-1$ character.
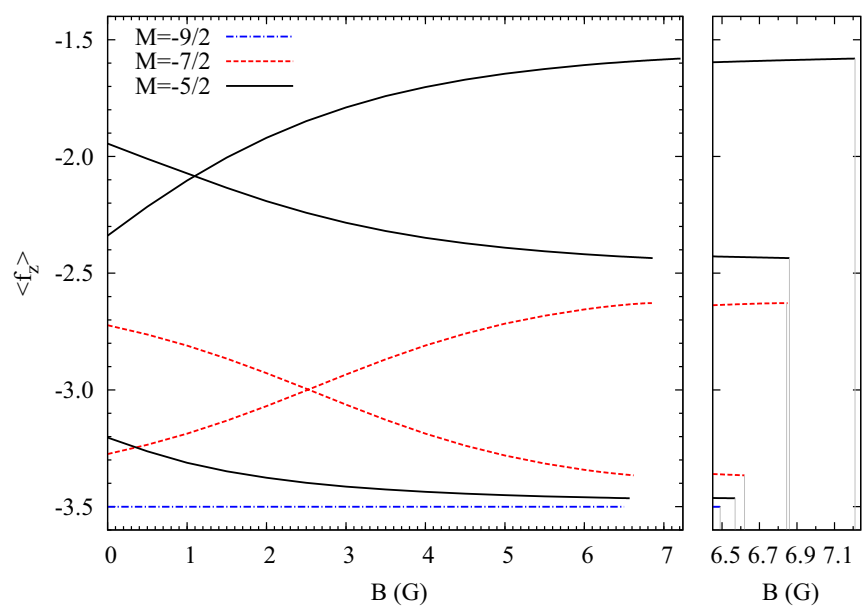

FIG. 3. Average value of the $z$ projection of the total internal spin $\vec{f}=\vec{f}_{a}+\vec{f}_{b}$ as a function of $B$ for $\ell=1$ bound states with total angular momentum projection $M$. The right panel presents a blowup for magnetic fields in the $6.4-7.2 \mathrm{G}$ range. 
Total rotational invariance and thus degeneracy with respect to $M$ is broken by the magnetic field, which introduces a coupling between states with different quantum number $F$ in subspaces of given $M$. As the magnetic field increases, the computed averaged projection $\left\langle f_{z}\right\rangle$ in the $\vec{B}$ direction converges for each of the six molecular states toward the large $B$ limit, that is, three levels with $m_{f}=-7 / 2$, two with $m_{f}=-5 / 2$, and one with $m_{f}=-3 / 2$.

This limit is, however, not fully reached when the boundstate energies cross threshold giving rise to resonance. We conclude that for the magnetic-field values of relevance for the resonances, the spin-spin perturbation is comparable with the Zeeman splitting. A treatment of the spin-spin interaction to first-order perturbation theory in subspaces spanned by states of given $m_{f}$ and $m=0, \pm 1$ is thus not appropriate in the present case. As a consequence, one cannot reach the usual conclusion that states with the same $|m|$ are degenerate, such approximate degeneracy being lifted by the perturbing effect of nearby states with different $m_{f}$.

Generalizing the argument above to other hyperfine combinations, we expect a multiplet of six resonances at low $B$ followed by a multiplet with eight resonances for the $|1,1\rangle+|9 / 2,-9 / 2\rangle$ channel. The $|1,1\rangle+|9 / 2,-7 / 2\rangle$ channel presents an eightfold multiplet at lower $B$ and a ninefold multiplet for larger $B$ values. The two remaining channels, $|1,1\rangle+|9 / 2,-5 / 2\rangle$ and $|1,1\rangle+|9 / 2,-3 / 2\rangle$, present two ninefold multiplets each. Some resonances are only weakly coupled and do not result in marked peaks in the elastic collision rate. More precisely, two molecular state crossings do not give rise to detectable features in the numerical elastic rate for the $|1,1\rangle+|9 / 2,-5 / 2\rangle$ channel (at 8.87 and at 24.80 $\mathrm{G})$. For the $|1,1\rangle+|9 / 2,-3 / 2\rangle$ channel, five molecular state crossings have no detectable effects on the numerical rate (at $11.35,11.95,32.00,35.74$, and $36.10 \mathrm{G}$ ). These features are, however, clearly seen in the inelastic probabilities.

We now propose an assignment of $p$-wave resonances in the MIT experiment. First of all, one may notice that the experimental spectrum only entails a subset of the predicted multiplets. Some features observed in the theoretical model (for instance, the pair near $6.85 \mathrm{G}$ or the one near $7.90 \mathrm{G}$ ) are strong but nearly overlapping, such that one can reasonably assume that they have not been resolved in the experiment. In such cases, for the assignment we only retain the strongest of the two features in the theoretical spectrum. Next, we affect the strongest elastic theoretical features to the experimental positions under the condition that the resulting splitting agrees with the experimental one. The procedure is successful in all cases, with the exception of the low- $B$ spectrum in the $|1,1\rangle+|9 / 2-5 / 2\rangle$ channel. Note, however, that the error given by MIT is relatively large for the $9.60 \mathrm{G}$ resonance.

A few theoretically weak resonances do not have an experimental counterpart, most likely because the corresponding experimental signature has been missed. The quality of our assignment, while still nonunivocal, strongly suggests that the dominant anisotropic interaction arises from the electronic spins [25].

\section{B. $\mathrm{Na}^{39} \mathrm{~K}$}

We continue our discussion with the most abundant potassium isotope, ${ }^{39} \mathrm{~K}$, a species for which cooling and Bose-Einstein condensation has traditionally proved to be difficult, yet finally achieved by different techniques [26,27].

TABLE IV. Same as Table II but for the isotopic pair $\mathrm{Na}^{39} \mathrm{~K}$. No experimental data are available for this system.

\begin{tabular}{|c|c|c|c|c|c|c|c|}
\hline $\mathrm{Na}^{39} \mathrm{~K}$ channel & $B_{\text {res }}(\mathrm{G})$ & $B_{\mathrm{ZC}}(\mathrm{G})$ & $r_{\mathrm{eff}}^{\mathrm{res}}\left(a_{0}\right)$ & $s_{\text {res }}$ & $a_{\mathrm{bg}}\left(a_{0}\right)$ & $\varepsilon\left(\mathrm{G}^{-1}\right)$ & $\Delta(\mathrm{G})$ \\
\hline$|1,1\rangle+|1,1\rangle$ & $\begin{array}{l}442.51 \\
536.00\end{array}$ & $\begin{array}{l}405.02 \\
533.72\end{array}$ & $\begin{array}{r}123.0 \\
-174.0\end{array}$ & $\begin{array}{l}4.00 \\
0.316\end{array}$ & -114.8 & $3.72 \times 10^{-4}$ & $\begin{array}{l}-36.9 \\
-2.27\end{array}$ \\
\hline$|1,1\rangle+|1,0\rangle$ & $\begin{array}{r}35.16 \\
356.21 \\
498.23 \\
606.51\end{array}$ & $\begin{array}{r}11.51 \\
355.45 \\
466.24 \\
603.13\end{array}$ & $\begin{array}{c}62.3 \\
-1.81 \times 10^{3} \\
118.0 \\
-88.2\end{array}$ & $\begin{array}{l}1.18 \\
0.0520 \\
3.35 \\
0.430\end{array}$ & & & \\
\hline$|1,0\rangle+|1,0\rangle$ & $\begin{array}{r}33.60 \\
107.97 \\
116.91\end{array}$ & $\begin{array}{l}19.39 \\
39.55\end{array}$ & $\begin{array}{r}-1.60 \times 10^{3} \\
117.0 \\
-969.0\end{array}$ & $\begin{array}{l}0.0586 \\
3.22 \\
0.0912\end{array}$ & $\begin{array}{r}258.0 \\
-569.0 \\
-10760\end{array}$ & & $\begin{array}{r}8.2 \\
-62.0 \\
0.18\end{array}$ \\
\hline$|1,1\rangle+|1,-1\rangle$ & $\begin{array}{l}116.98 \\
422.51 \\
566.06 \\
688.63\end{array}$ & $\begin{array}{l}421.86 \\
539.80 \\
685.97\end{array}$ & $\begin{array}{c}-3.85 \times 10^{4} \\
-2.02 \times 10^{3} \\
111.0 \\
-170.0\end{array}$ & $\begin{array}{l}0.00264 \\
0.0469 \\
2.74 \\
0.320\end{array}$ & & & \\
\hline$|1,-1\rangle+|1,0\rangle$ & 56.31 & 54.70 & $-3.04 .0 \times 10^{4}$ & 0.00334 & & & \\
\hline$|1,0\rangle+|1,-1\rangle$ & 158.18 & & 141.0 & 14.1 & & & \\
\hline$|1,1\rangle+|2,-2\rangle$ & $\begin{array}{l}498.48 \\
648.26\end{array}$ & $\begin{array}{l}498.22 \\
627.58\end{array}$ & $\begin{array}{c}-4.93 \times 10^{3} \\
102.0\end{array}$ & $\begin{array}{l}0.0201 \\
2.20\end{array}$ & & & \\
\hline$|1,-1\rangle+|1,-1\rangle$ & $\begin{array}{r}2.01 \\
241.40\end{array}$ & 75.71 & $\begin{array}{l}142.0 \\
107.0\end{array}$ & $\begin{array}{l}15.2 \\
2.47\end{array}$ & $\begin{array}{r}-183.7 \\
-62.6\end{array}$ & $1.86 \times 10^{-2}$ & $\begin{array}{l}63.2 \\
53.0\end{array}$ \\
\hline$|1,0\rangle+|2,-2\rangle$ & $\begin{array}{l}357.96 \\
657.18\end{array}$ & $\begin{array}{l}357.10 \\
599.44\end{array}$ & $\begin{array}{c}-9.66 \times 10^{3} \\
132.0\end{array}$ & $\begin{array}{l}0.0104 \\
6.30\end{array}$ & & & \\
\hline
\end{tabular}



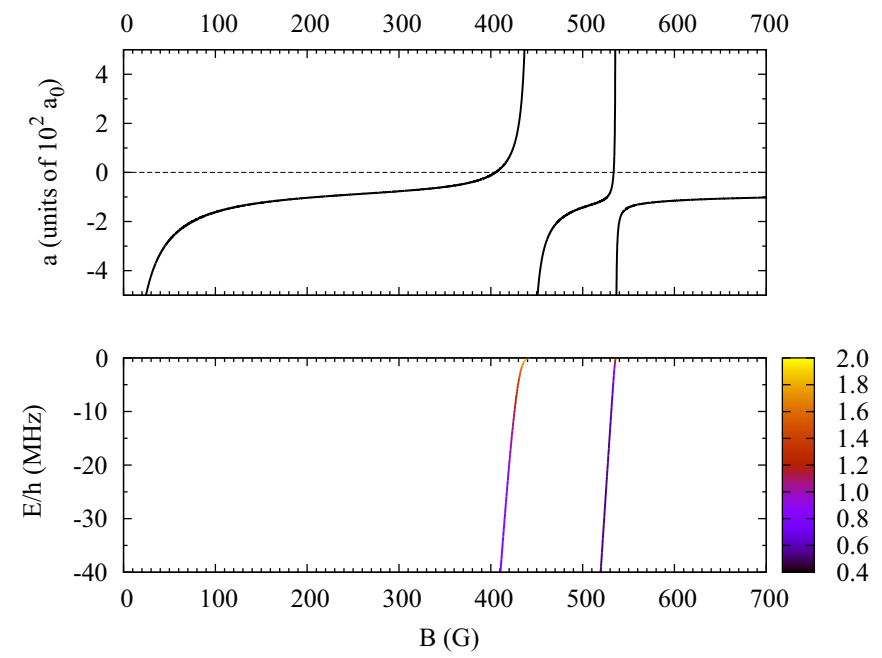

FIG. 4. Scattering length $a$ as a function of the magnetic field $B$ for $\mathrm{Na}|1,1\rangle+{ }^{39} \mathrm{~K}|1,1\rangle s$-wave collisions (top panel). Corresponding molecular energy levels as a function of $B$ are shown in the lower panel. The density code denotes the average spin $\left\langle\vec{S}^{2}\right\rangle$ of the molecule.

We provide results for a series of hyperfine states. Our data can therefore be useful in order to interpret collision data in a pure spin or in the case of partial polarization of the sample. Toward that end, calculations of the $s$-wave scattering length are performed for different values of the conserved projection of the total angular momentum, $M$. Table IV summarizes the $s$-wave resonances found for $\mathrm{Na}^{39} \mathrm{~K}$ for a magnetic field up to $1000 \mathrm{G}$. We report the positions of the 21 poles observed in the scattering length, $B_{\text {res }}$, as well as the 17 zero-crossing fields, $B_{\mathrm{ZC}}$. Note that no zero-crossing exists for $\mathrm{Na}|1,0\rangle+{ }^{39} \mathrm{~K}|1,-1\rangle$ collisions and that a single one at $B_{\mathrm{ZC}}=75.71 \mathrm{G}$ is present for the $\mathrm{Na}|1,-1\rangle{ }^{39} \mathrm{~K}|1,-1\rangle$ channel. Since we include only $s$-waves, possible narrow spinspin resonances due, for instance, to $s \rightarrow d$ wave couplings are not reproduced by the model. The incoming state for the collision is systematically taken to be the lowest-energy state with the given $M$ at magnetic field intensity $B$. Note that in general this state may decay by inelastic spin-spin processes if $\ell>0$ states were included in the basis. However, these processes will tend to be slow except very close to resonance, and they are neglected for computational simplicity. Figures 4,5 , and 6 provide the scattering length as well as the molecular energies for three of the nine studied channels. Numerical data are available upon request.

As in the boson-fermion case we choose to parametrize the field-dependent $s$-wave scattering length by the unique expression Eq. (4) over a magnetic-field range of $\pm 4 \Delta$ around the resonance, and we compute the resonance length in order to assess the resonance strength. For overlapping resonances, a unique $a_{\mathrm{bg}}$ and $\epsilon$ values are given, whereas for isolated resonances we give a local $a_{\text {bg }}$ and $\epsilon$ when the latter is nonvanishing. We achieve the sufficient required accuracy (below 5\%, as for the ${ }^{40} \mathrm{~K}$ isotope) for three of the nine initial channels considered. Many combinations are found to be not well described by Eq. (4), in particular in the presence of energetically degenerate channels that give rise to characteristic threshold singularities [28].
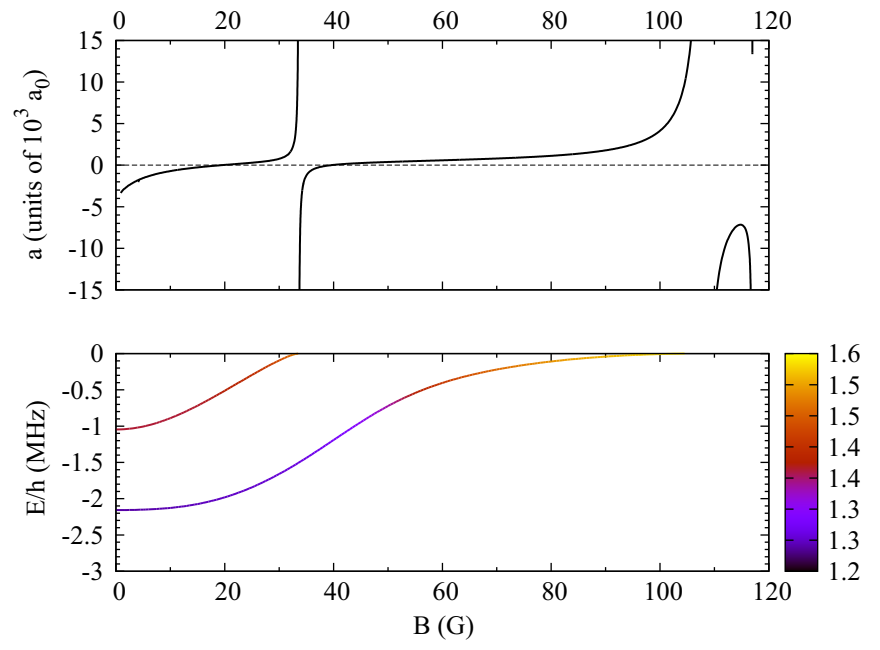

FIG. 5. Same as Fig. 4 but for $\mathrm{Na}|1,0\rangle+{ }^{39} \mathrm{~K}|1,0\rangle$ collisions.

Both open $s_{\text {res }} \gg 1$ and closed $s_{\text {res }} \ll 1$ channel dominated resonances are available in suitable hyperfine combinations. A particularly interesting feature is the one near $442 \mathrm{G}$ for collisions in the absolute hyperfine ground state $\mid f_{a}=$ $\left.1, m_{a}=1\right\rangle+\left|f_{b}=1, m_{b}=1\right\rangle$, which is strictly stable under two-body inelastic collisions and open-channel dominated; see also Fig. 4. Its large magnetic width $|\Delta|=36.9 \mathrm{G}$ should allow one to tune $a$ to desired values with high accuracy and thus possibly to explore the quantum phases predicted in free space and under optical-lattice confinement for a variety of geometries [29-31]. Also note that for vanishing $B$, the scattering length $a$ is negative and very large in magnitude, $|a|>5 \times 10^{3} a_{0}$, a feature related to the presence of a virtual state with positive energy. Variation with $B$ of the position of the virtual state results in the rapid variation of $a$ with magnetic field observed for small $B$.

The known FRs for $\mathrm{Na}+\mathrm{Na}$ collisions in the ground state are located at large fields $B>800 \mathrm{G}$ [32] in a region where $a_{\mathrm{KK}}$ and $a_{\mathrm{NaK}}$ present regular nonresonant behavior. (For reference,
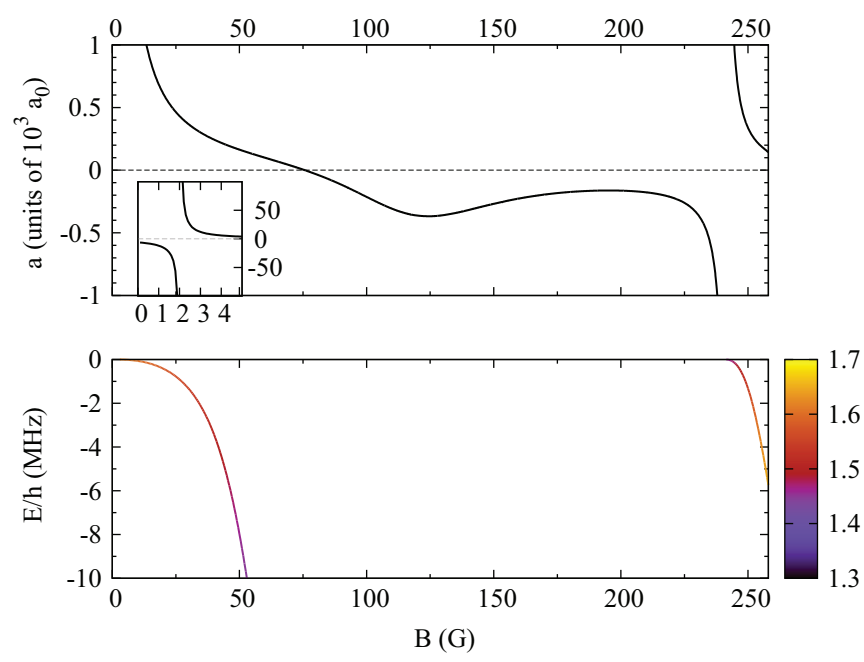

FIG. 6. Same as Fig. 4 but for $\mathrm{Na}|1,-1\rangle+{ }^{39} \mathrm{~K}|1,-1\rangle$ collisions. The inset shows the behavior of $a$ at low field on a larger vertical scale. 

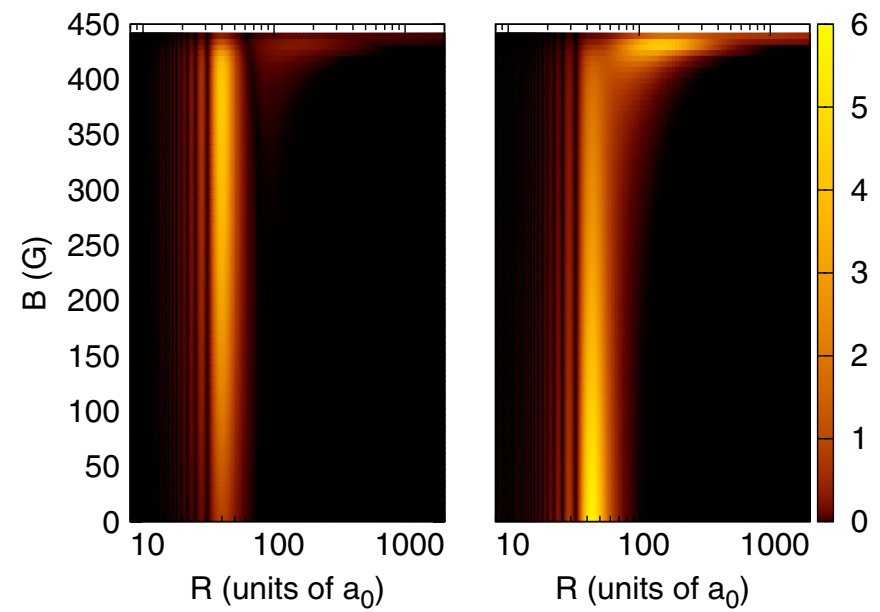

FIG. 7. The $S=0$ (left panel) and $S=1$ (right panel) electron spin components in arbitrary units of last-below-threshold $\ell=0$ multichannel wave function with $M=2$ as a function of the magnetic field $B$ for the $\mathrm{Na}^{39} \mathrm{~K}$ dimer.

$a_{\mathrm{KK}} \simeq-35 a_{0}$ and $a_{\mathrm{NaK}} \simeq-90 a_{0}$.) Tuning of the interspecies scattering length can be used to increase the cross section for sympathetic cooling, for instance to $\operatorname{cool}{ }^{39} \mathrm{~K}$ by thermal contact with ultracold Na. A comparison of Fig. 4 of this paper with Fig. 4 of Ref. [33] shows that at the field $B=$ $395.2 \mathrm{G}$ at which ${ }^{39} \mathrm{~K}$ has been condensed [34], the $a_{\mathrm{NaK}}$ is slightly negative. A double BEC of sodium and potassium will thus be miscible and stable against collapse [35]. Moreover, if the double condensate is adiabatically loaded in an optical lattice, the attractive character of the $\mathrm{NaK}$ effective interaction will favor the loading of $\mathrm{Na}$ and $\mathrm{K}$ pairs at the lattice cells. This should be an advantageous starting point to associate Feshbach molecules and thus implement STIRAP schemes to form ultracold molecules in the absolute ground state.

Indeed, as compared to the boson-fermion case of Sec. III A, one can verify from the $\left\langle\vec{S}^{2}\right\rangle$ given by the color code in the

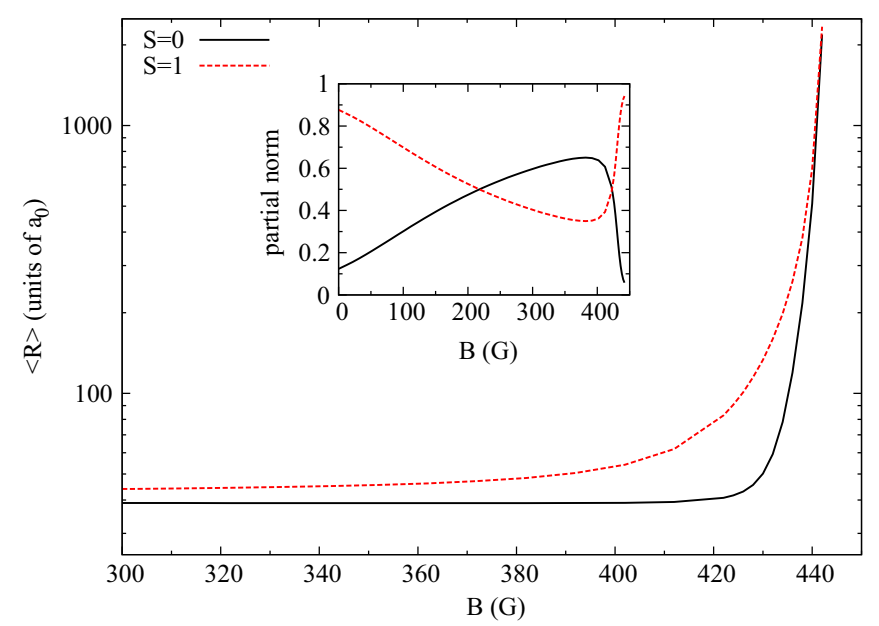

FIG. 8. Average distance $\langle R\rangle$ and partial singlet/triplet norms (defined in the text) of the last-below-threshold $\ell=0$ multichannel wave function with $M=2$ as a function of the magnetic field $B$ for the $\mathrm{Na}^{39} \mathrm{~K}$ dimer.
TABLE V. Theoretical magnetic-field locations $B_{\text {res }}$ of $p$-wave FR for $\mathrm{Na}^{39} \mathrm{~K}$ for projection of total angular momentum $M$ in different hyperfine atomic channels. Calculations are performed at a collision energy of $1 \mu \mathrm{K}$.

\begin{tabular}{lcc}
\hline \hline $\mathrm{Na}^{39} \mathrm{~K}$ channel & $M$ & $B_{\text {res }}(\mathrm{G})$ \\
\hline$|1,1\rangle+|1,1\rangle$ & 1 & 242.83 \\
& 1 & 260.19 \\
& 1 & 354.02 \\
& 1 & 370.58 \\
& 1 & 395.53 \\
$|1,1\rangle+|1,1\rangle$ & 1 & 462.21 \\
& 1 & 491.27 \\
& 1 & 529.67 \\
& 2 & 242.68 \\
& 2 & 353.95 \\
& 2 & 370.82 \\
& 2 & 438.27 \\
& 2 & 461.84 \\
& 2 & 491.68 \\
& 3 & 354.01 \\
& 3 & 437.99 \\
& 3 & 462.21 \\
\hline \hline
\end{tabular}

lower panel of Fig. 4 that the situation here is favorable, since the molecule presents hyperfine-induced singlet-triplet mixing even far from dissociation. Beyond the average spin character, we also represent in Fig. 7 the details of the singlet and triplet components of the coupled wave function, defined as $\Psi_{0}=$ $\hat{P}_{0} \Psi$ and $\Psi_{1}=\hat{P}_{1} \Psi$, with $\hat{P}_{0,1}$ the projectors on the $S=0$ and 1 subspaces, respectively. Interestingly, the $S=0$ amplitude reaches its maximum right before the resonance, at $B \approx 400 \mathrm{G}$. Most importantly, Fig. 7 shows that $\Psi_{0}$ maintains a short-range character with maximum amplitude for $R \approx 40 a_{0}$.

Such short-range character is confirmed quantitatively in Fig. 8 by calculations of the average distances $\langle R\rangle_{0,1}=$

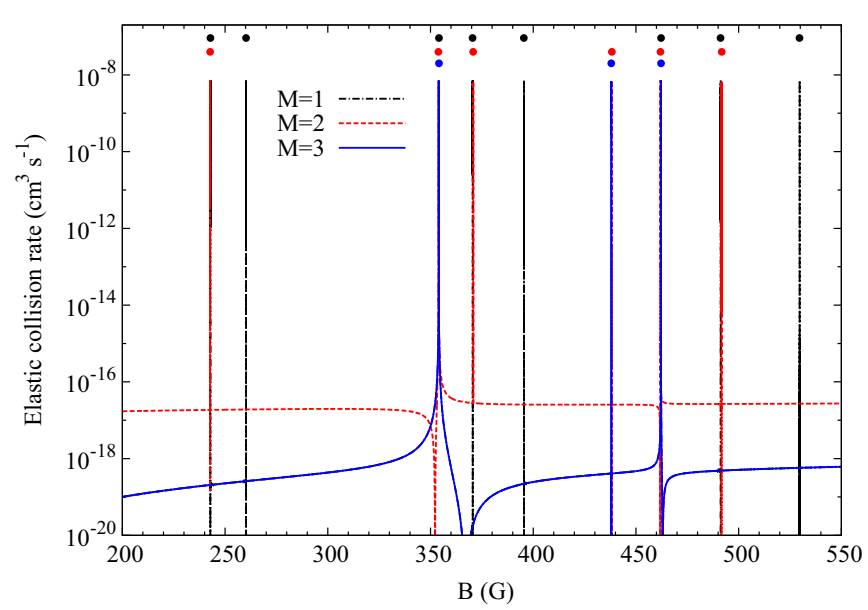

FIG. 9. Elastic collision rate computed at a collision energy of $1 \mu \mathrm{K}$ as a function of the magnetic field $B$ for $\mathrm{Na}|1,1\rangle+{ }^{39} \mathrm{~K}|1,1\rangle$ $p$-wave collisions. The dots materialize the occurrence of maxima in the rate for each projection $M$. 
$\left\langle\Psi_{0,1}|\hat{R}| \Psi_{0,1}\right\rangle$. Moreover, the partial norms $\left|\left\langle\Psi_{0,1} \mid \Psi_{0,1}\right\rangle\right|^{2}$ depicted in the figure show that the singlet admixture is significant in the region of interest. Analyses of the electronic excited-state structure of $\mathrm{NaK}$ and of the corresponding Frank-Condon factor for transfer of the Feshbach molecule to the excited state is beyond the scope of this work. However, Ref. [14] finds relatively favorable Frank-Condon factors in the case of Bose-Fermi Feshbach molecules, which present a similar spatial extent to that of the present bosonic ones but with a significantly smaller singlet component. We expect, therefore, that suitable excited states can be found to implement an efficient two-photon transfer in the present case.

Let us now consider collisions for atoms in the first excited hyperfine level $|1,0\rangle$ reproduced in Fig. 5. Similar to the case of the absolute ground state, a large negative scattering length rapidly varying with $B$ is predicted at low magnetic fields. A point of nonanalyticity is expected at a magnetic field $B \simeq 117$
$\mathrm{G}$ as the $|1,0\rangle+|1,0\rangle$ and $|1,1\rangle+|1,-1\rangle$ channels become degenerate. It is interesting to observe here that the expected cusp in the elastic-scattering matrix element is accompanied by poles in $a$ occurring right before (after) the degeneracy point in the $|1,0\rangle+|1,0\rangle(|1,1\rangle+|1,-1\rangle)$ channel, a peculiar effect stemming from the interplay between channel degeneracy and Feshbach physics; see Fig. 5 and Table IV.

The case of $|1,-1\rangle+|1,-1\rangle$ collisions is shown in Fig. 6 and is particularly relevant for the applications since $|1,-1\rangle$ is the lowest magnetically trappable atomic state of ${ }^{39} \mathrm{~K}$ and $\mathrm{Na}$ at low $B$. Moreover, below $259 \mathrm{G}$ the $\mathrm{NaK}|1,-1\rangle+|1,-1\rangle$ combination is stable under $s$-wave collisions since it is the lowest hyperfine state with $m_{f}=-2$. Note that due to the presence of a Feshbach resonance at very low magnetic fields $B=2 \mathrm{G}$, the scattering zero-field length is negative and very large in magnitude (see the inset of Fig. 6). Bose-Einstein condensation has been achieved in this hyperfine level using magnetic tuning of $a$ to suitable values [26,27]. Interestingly,

TABLE VI. Same as Table IV but for $\mathrm{Na}^{41} \mathrm{~K}$.

\begin{tabular}{|c|c|c|c|c|c|c|c|}
\hline $\mathrm{Na}^{41} \mathrm{~K}$ channel & $B_{\text {res }}(\mathrm{G})$ & $B_{\mathrm{ZC}}(G)$ & $r_{\text {eff }}^{\text {res }}\left(a_{0}\right)$ & $s_{\text {res }}$ & $a_{\mathrm{bg}}\left(a_{0}\right)$ & $\varepsilon\left(\mathrm{G}^{-1}\right)$ & $\Delta(\mathrm{G})$ \\
\hline \multirow[t]{5}{*}{$|1,1\rangle+|1,1\rangle$} & 20.90 & 20.90 & $-6.55 \times 10^{6}$ & $1.56 \times 10^{-5}$ & \multirow[t]{5}{*}{334.80} & \multirow[t]{5}{*}{$-1.03 \times 10^{-4}$} & $3.57 \times 10^{-5}$ \\
\hline & 51.23 & 51.30 & $-2.76 \times 10^{3}$ & $3.52 \times 10^{-2}$ & & & $7.10 \times 10^{-2}$ \\
\hline & 73.35 & 77.97 & 85.2 & 1.60 & & & 4.59 \\
\hline & 470.08 & 476.41 & 104. & 2.25 & & & 6.32 \\
\hline & $\begin{array}{r}531.59 \\
(-235.65)\end{array}$ & 532.16 & -458.0 & $1.68 \times 10^{-1}$ & & & $\begin{array}{c}5.63 \times 10^{-1} \\
\left(6.89 \times 10^{1}\right)\end{array}$ \\
\hline \multirow[t]{8}{*}{$|1,1\rangle+|1,0\rangle$} & 33.26 & 33.26 & $-6.18 \times 10^{5}$ & $1.66 \times 10^{-4}$ & & & \\
\hline & 35.53 & 35.53 & $-3.70 \times 10^{7}$ & $2.76 \times 10^{-6}$ & & & \\
\hline & 66.48 & 66.61 & $-1.58 \times 10^{3}$ & $5.93 \times 10^{-2}$ & & & \\
\hline & 87.53 & 90.94 & 58.1 & 1.12 & & & \\
\hline & 165.58 & 165.60 & $-4.72 \times 10^{4}$ & $2.16 \times 10^{-3}$ & & & \\
\hline & 453.37 & 453.37 & $-1.03 \times 10^{5}$ & $9.88 \times 10^{-4}$ & & & \\
\hline & 499.41 & 506.39 & 108.0 & 2.50 & & & \\
\hline & 566.30 & 567.17 & -244.0 & $2.60 \times 10^{-1}$ & & & \\
\hline$|1,0\rangle+|1,0\rangle$ & 35.05 & 35.05 & $-5.67 \times 10^{6}$ & $1.80 \times 10^{-5}$ & 246.1 & $9.71 \times 10^{-4}$ & $8.08 \times 10^{-4}$ \\
\hline \multirow[t]{8}{*}{$|1,1\rangle+|1,-1\rangle$} & 63.46 & 63.48 & $-2.87 \times 10^{4}$ & $3.55 \times 10^{-3}$ & & & \\
\hline & 72.53 & 72.53 & $-8.85 \times 10^{5}$ & $1.16 \times 10^{-4}$ & & & \\
\hline & 106.20 & 107.69 & -77.5 & $4.51 \times 10^{-1}$ & & & \\
\hline & 183.36 & 183.36 & $-1.25 \times 10^{7}$ & $8.17 \times 10^{-6}$ & & & \\
\hline & 370.11 & 370.11 & $-1.20 \times 10^{6}$ & $8.51 \times 10^{-5}$ & & & \\
\hline & 481.53 & 481.53 & $-1.34 \times 10^{4}$ & $7.62 \times 10^{-4}$ & & & \\
\hline & 531.87 & 539.42 & 111.0 & 2.70 & & & \\
\hline & 604.62 & 605.36 & -309.0 & $2.23 \times 10^{-1}$ & & & \\
\hline \multirow[t]{3}{*}{$|1,0\rangle+|1,-1\rangle$} & 66.97 & 66.98 & $-8.54 \times 10^{5}$ & $1.20 \times 10^{-4}$ & & & \\
\hline & 129.37 & 129.60 & $-1.98 \times 10^{3}$ & $4.81 \times 10^{-2}$ & & & \\
\hline & 149.33 & 149.32 & $-2.68 \times 10^{5}$ & $3.82 \times 10^{-4}$ & & & \\
\hline \multirow[t]{5}{*}{$|1,1\rangle+|2,-2\rangle$} & 156.22 & 156.22 & $-1.16 \times 10^{6}$ & $8.84 \times 10^{-5}$ & & & \\
\hline & 209.92 & 209.93 & $-5.51 \times 10^{4}$ & $1.85 \times 10^{-3}$ & & & \\
\hline & 391.25 & 391.25 & $-1.26 \times 10^{6}$ & $8.13 \times 10^{-5}$ & & & \\
\hline & 512.63 & 512.63 & $-3.84 \times 10^{5}$ & $2.66 \times 10^{-4}$ & & & \\
\hline & 567.79 & 575.74 & 113.0 & 2.82 & & & \\
\hline$|1,-1\rangle+|1,-1\rangle$ & 137.27 & 137.27 & $-4.13 \times 10^{5}$ & $2.48 \times 10^{-4}$ & 212.75 & 0 & $-2.43 \times 10^{-3}$ \\
\hline \multirow[t]{4}{*}{$|1,0\rangle+|2,-2\rangle$} & 146.65 & 146.65 & $-9.73 \times 10^{5}$ & $1.05 \times 10^{-4}$ & & & \\
\hline & 245.19 & 252.66 & 91.4 & 1.77 & & & \\
\hline & 500.76 & 500.82 & $-9.51 \times 10^{3}$ & $1.06 \times 10^{-2}$ & & & \\
\hline & 601.15 & 606.56 & 92.9 & 1.81 & & & \\
\hline
\end{tabular}



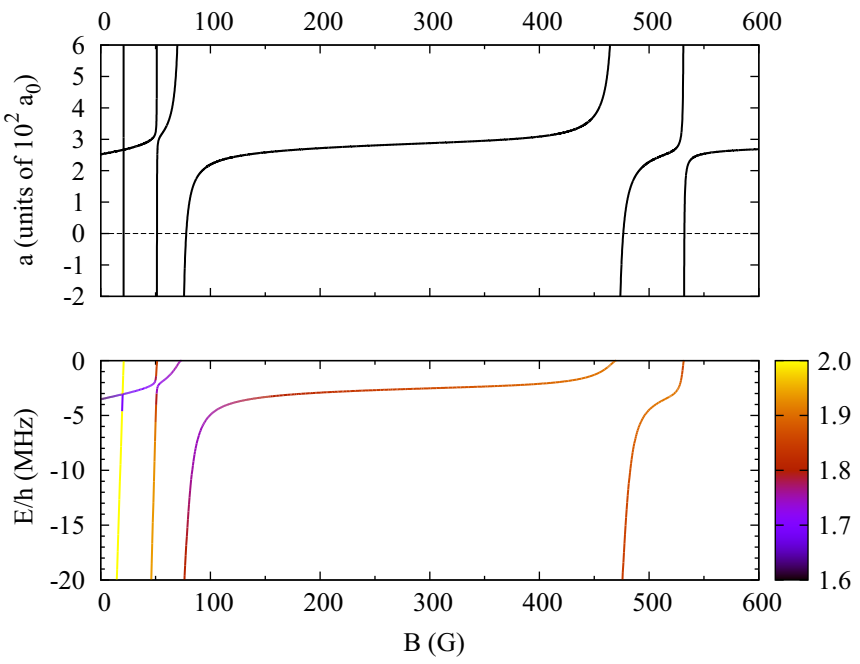

FIG. 10. Scattering length $a$ as a function of the magnetic field $B$ for $\mathrm{Na}|1,1\rangle+{ }^{41} \mathrm{~K}|1,1\rangle$ collisions (top panel) in the $s$ wave. Corresponding molecular energy levels as a function of $B$ are shown in the lower panel. The density code denotes the average spin $\left\langle\vec{S}^{2}\right\rangle$ of the molecule.

Fig. 5 of Ref. [33] shows that for a magnetic field between the two homonuclear $\mathrm{K}$ resonances located in $|1,-1\rangle$ at about 33 and $163 \mathrm{G}$, the $a_{\mathrm{KK}}$ is positive, thus ensuring the stability of a $\mathrm{K}$ condensate. In the same magnetic-field region, $a_{\mathrm{NaK}}$ varies from being large and positive to large and negative, allowing one to explore the phase diagram of a quantum degenerate $\mathrm{NaK}$ mixture as a function of the mutual interaction strength.

To conclude our analysis for this isotope, we provide in Table $\mathrm{V}$ the spectrum of $p$-wave resonances, limiting ourselves to the absolute ground state. As in the case of the bosonfermion mixtures [12], such resonances can be experimentally observable even at ultracold temperatures. Figure 9 shows the elastic collision rate for different $M$ projections presenting a rich spectrum with nearby peaks of multiplicities 3,2 , and 1. Closer inspection shows that triply degenerate peaks are the usual doublets [24], with the peaks arising from spin-spin-induced mixing of $m_{f}=2, m= \pm 1$ states being nearly degenerate and slightly shifted with respect to the $m_{f}=$ $2, m=0$ peak. Larger multiplicities such as those in Fig. 2 are not observed here since $p$-wave resonances occur at larger magnetic fields; see the discussion in Sec. III A. According to the value of $M$ in Fig. 9, doubly degenerate components arise from coupling to states with $m_{f}=1, m=0,1$ or to states with $m_{f}=3, m=-1,0$. Finally, all singly degenerate levels in the figure are due to coupling with $m_{f}=0, m=1$.

\section{C. $\mathrm{Na}^{41} \mathrm{~K}$}

We now provide numerical data for the other bosonic pair $\mathrm{Na}^{41} \mathrm{~K}$. Let us first recall that ${ }^{41} \mathrm{~K}$ has been brought to BoseEinstein condensation using $\mathrm{Rb}$ as a coolant, or more recently by direct evaporation [36]. Resonances exist for collisions in different hyperfine states with magnetic widths of several G; see Table VI.
Such broad resonances are essentially open-channel dominated, with a resonance strength of $s_{\text {res }} \lesssim 3$. Several closedchannel dominated features are also readily available in each hyperfine channel we studied. A distinctive feature of $\mathrm{Na}^{41} \mathrm{~K}$ is the large and positive $a_{\mathrm{bg}}$ for all the hyperfine combinations. The parametrization Eq. (4) for overlapping resonances is used for the absolute ground state, where it is found to be accurate only if an artificial pole is added in Eq. (4) at negative $B$. Such a pole mimics the effect of a virtual state, i.e., a quasibound state located at positive energy and that would give a resonance at negative values of $B$. The position obtained through the fitting procedure is given in Table VI in parentheses to distinguish from physical poles of $a$. The corresponding scattering length $a$ is given in the top panel of Fig. 10 .

Both ${ }^{41} \mathrm{~K}$ and $\mathrm{Na}$ homonuclear resonances in $|1,1\rangle+|1,1\rangle$ are narrow and quite sparse. A combination of the present and the magnetic spectra in Refs. [33] and [32] for $\mathrm{K}$ and $\mathrm{Na}$, respectively, shows that homonuclear and heteronuclear resonances take place at well-separated locations. Note that the large $a_{\mathrm{bg}}$ for $\mathrm{NaK}$ and the nonresonant values of order $50 a_{0}$ for both $\mathrm{Na}$ and ${ }^{41} \mathrm{~K}$ imply that two Bose-Einstein condensates will tend to phase-separate. However, the heteronuclear resonances can be used to reduce or even change the sign of $a_{\mathrm{NaK}}$ so as to favor miscibility and eventually the realization of overlapping quantum gases of $\mathrm{Na}$ and $\mathrm{K}$ in free space or in optical lattices.

Let us now discuss the magnetic association of $\mathrm{Na}$ and $\mathrm{K}$ atoms when they are prepared in the respective ground hyperfine levels. A calculation of the quantum average $\langle\vec{S}\rangle^{2}$ depicted as density code in the lower panel of Fig. 10 readily shows that resonances arise from states with dominant triplet character. Note that the large background scattering length implies the existence of a molecular level close to the dissociation threshold; see the lower panel of Fig. 10. Let us consider performing magnetic association near the two broadest FRs. Based on our data, three routes can be envisioned, although they still present drawbacks.
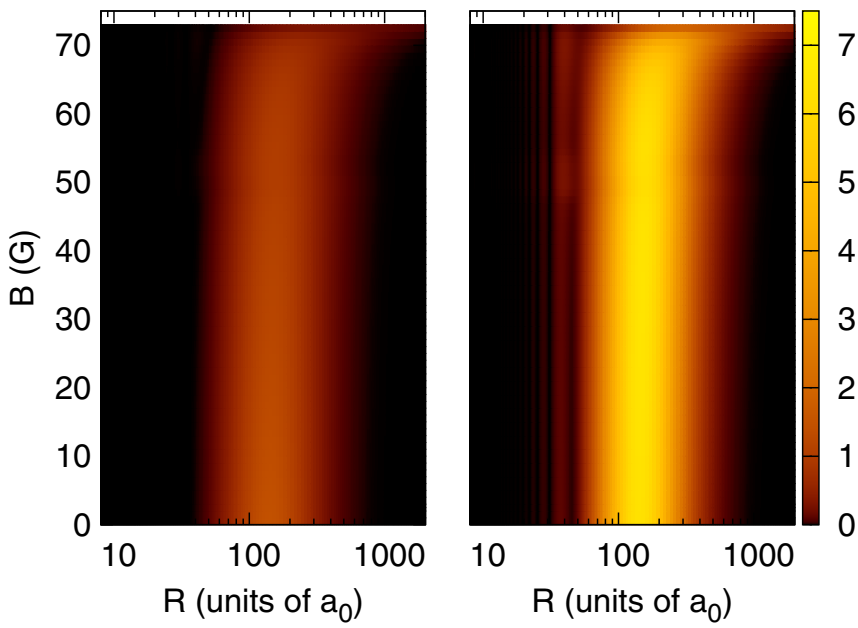

FIG. 11. The $S=0$ (left panel) and $S=1$ (right panel) electron spin components using the same arbitrary units of Fig. 7 of the $\ell=0$ multichannel wave function with $M=2$ as a function of the magnetic field $B$ for the $\mathrm{Na}^{41} \mathrm{~K}$ dimer. The molecule is created at $73.35 \mathrm{G}$ and the molecular state is followed diabatically with decreasing $B$. 


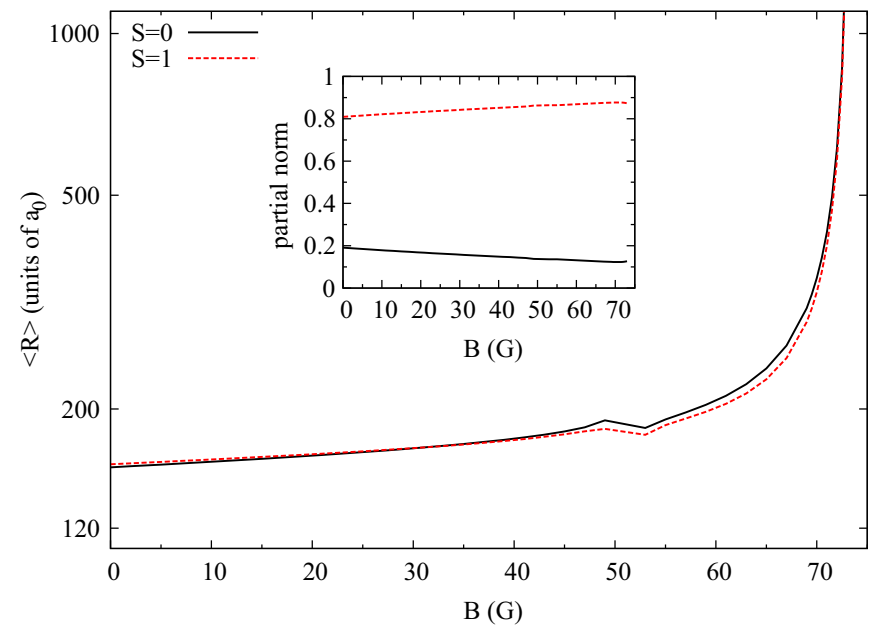

FIG. 12. Average distance $\langle R\rangle$ and partial singlet/triplet norms (defined in the text) of the last-below-threshold $\ell=0$ multichannel wave function with $M=2$ as a function of the magnetic field $B$ for the $\mathrm{Na}^{39} \mathrm{~K}$ dimer. The molecular state is followed diabatically as for Fig. 11.

If molecules are formed at the $73 \mathrm{G} \mathrm{FR}$ and molecular curve crossings are swept through diabatically, one stays in the "background" weakest bound molecular level. Unfortunately, as shown in Figs. 11 and 12, the state has long-range character with $\langle R\rangle_{0,1} \gtrsim 100 a_{0}$.

Therefore, in spite of the sufficient singlet character predicted in Fig. 12, poor overlap is expected with the excited molecular states. Note that since quantum numbers of this background state are essentially atomic ones or Hund's case (e), the projections $\Psi_{0}$ and $\Psi_{1}$ on the Hund's case (b) spincoupled basis in Fig. 11 have virtually identical spatial profiles.

An alternative route consists in following adiabatically the entrance state through the first avoided crossing near $50 \mathrm{G}$. As shown in the lower panel of Fig. 10, this leads, however,
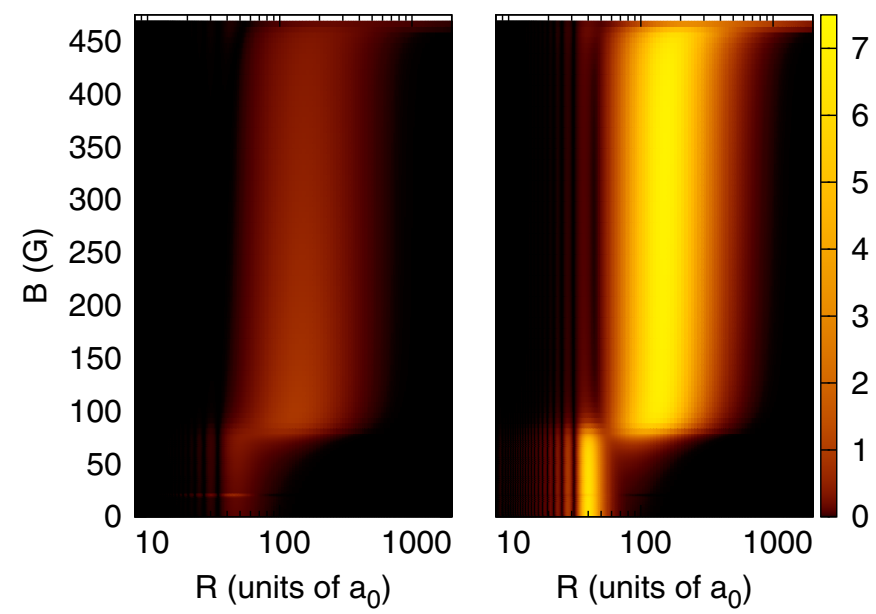

FIG. 13. The $S=0$ (left panel) and $S=1$ (right panel) electron spin components using the same arbitrary units of Fig. 7 of the $\ell=0$ multichannel wave function with $M=2$ as a function of the magnetic field $B$ for the $\mathrm{Na}^{41} \mathrm{~K}$ dimer. The molecule is created at $470 \mathrm{G}$ and the molecular state is followed adiabatically with decreasing $B$.

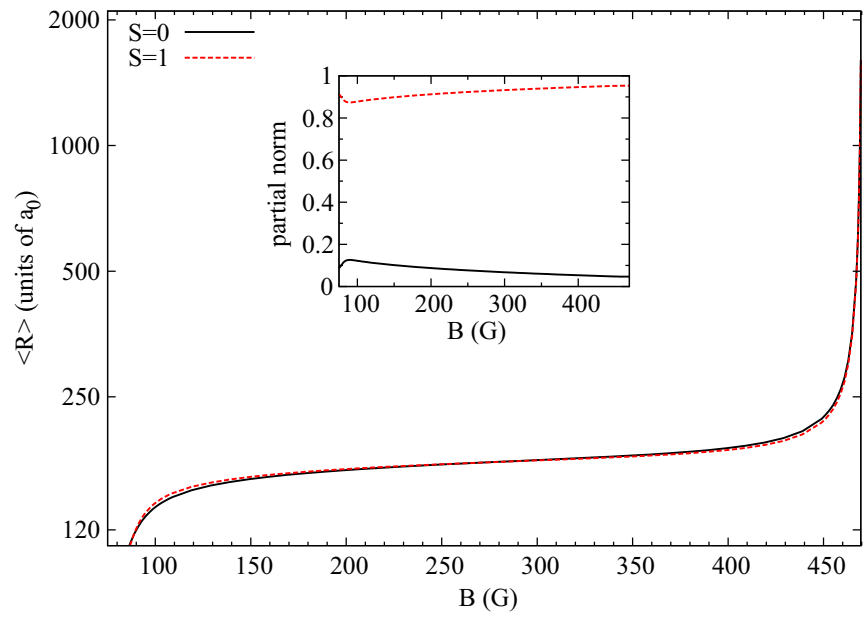

FIG. 14. Average distance $\langle R\rangle$ and partial singlet/triplet norms (defined in the text) of the last-below-threshold $\ell=0$ multichannel wave function with $M=2$ as a function of the magnetic field $B$ for the $\mathrm{Na}^{39} \mathrm{~K}$ dimer. The molecular state is followed adiabatically as for Fig. 13.

to the formation of a molecule with a relatively poor singlet admixture.

If one uses the broad resonance at $470 \mathrm{G}$ as an entrance gate, a long magnetic-field sweep down to $B \sim 60 \mathrm{G}$ would be needed before a small $\langle R\rangle_{0}$ is attained, as can be inferred from Fig. 13 and the main panel of Fig. 14. However, the inset in the latter figure indicates that molecule shrinking also

TABLE VII. Same as Table V but for $\mathrm{Na}^{41} \mathrm{~K}$.

\begin{tabular}{|c|c|c|}
\hline $\mathrm{Na}^{41} \mathrm{~K}$ channel & $M$ & $B_{\text {res }}(\mathrm{G})$ \\
\hline \multirow[t]{13}{*}{$|1,1\rangle+|1,1\rangle$} & 1 & 17.82 \\
\hline & 1 & 20.72 \\
\hline & 1 & 31.47 \\
\hline & 1 & 40.94 \\
\hline & 1 & 133.49 \\
\hline & 1 & 377.46 \\
\hline & 1 & 386.90 \\
\hline & 1 & 418.20 \\
\hline & 1 & 426.43 \\
\hline & 1 & 438.89 \\
\hline & 1 & 466.48 \\
\hline & 1 & 478.98 \\
\hline & 1 & 495.58 \\
\hline \multirow[t]{9}{*}{$|1,1\rangle+|1,1\rangle$} & 2 & 17.79 \\
\hline & 2 & 20.47 \\
\hline & 2 & 41.89 \\
\hline & 2 & 377.84 \\
\hline & 2 & 417.19 \\
\hline & 2 & 427.37 \\
\hline & 2 & 453.54 \\
\hline & 2 & 465.11 \\
\hline & 2 & 480.30 \\
\hline \multirow[t]{4}{*}{$|1,1\rangle+|1,1\rangle$} & 3 & 17.84 \\
\hline & 3 & 418.19 \\
\hline & 3 & 452.11 \\
\hline & 3 & 466.47 \\
\hline
\end{tabular}




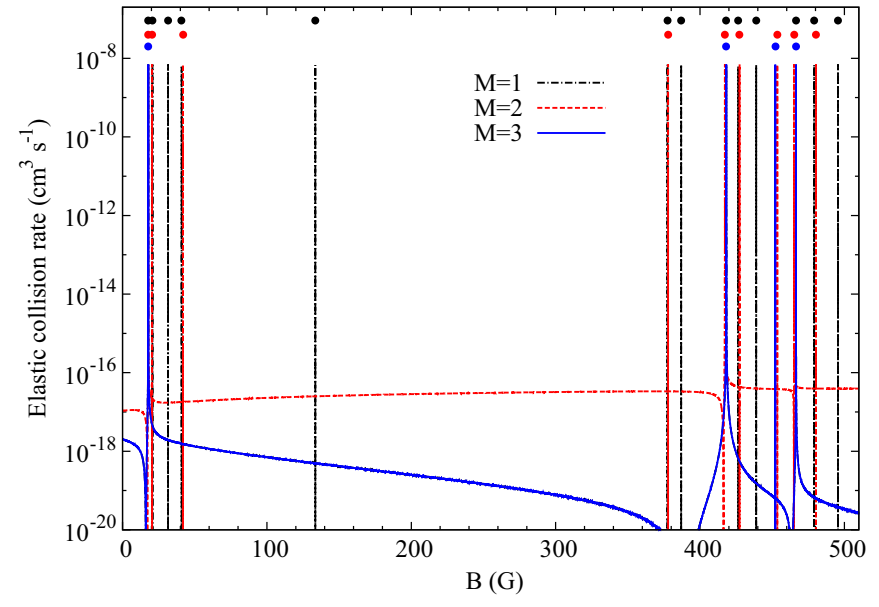

FIG. 15. Elastic rate computed at a collision energy of $1 \mu \mathrm{K}$ as a function of the magnetic field $B$ for $\mathrm{Na}|1,1\rangle+{ }^{41} \mathrm{~K}|1,1\rangle p$-wave collisions. The color dots above each peak display the occurrence of maxima in the rate for each projection $M$.

corresponds to a drop in the singlet character, thus requiring a compromise. To draw firmer conclusions, a detailed analysis of the excited states will be needed.

Finally, as illustrated in Fig. 15, our model predicts a series of $p$-wave Feshbach resonances at both weak and strong magnetic fields in the absolute ground state. Table VII confirms as expected that multiplet splittings at small $B$ are "anomalous" in the same sense as for a boson-fermion pair, whereas as in $\mathrm{Na}^{39} \mathrm{~K}$ they follow standard patterns at large $B$. Experimental observation of the corresponding magnetic spectra would provide a valuable piece of information to confirm the accuracy of our model for the $\ell>0$ collision in this boson-boson mixture.

\section{CONCLUSIONS}

We have presented an extensive compendium of the groundstate scattering properties of isotopic $\mathrm{NaK}$ mixtures in an external magnetic field. Our results complement existing theory and experimental data on the boson-fermion pair $\mathrm{Na}^{40} \mathrm{~K}$. The Feshbach resonance locations and strengths we predict for the boson-boson pairs should be of major interest for experiments in which control of the atom-atom interaction is a requirement. Our spin-resolved analysis of Feshbach molecules also provides an important piece of information for designing magnetoassociation and the two-photon transfer scheme of Feshbach molecules to the absolute rovibrational ground state.

\section{ACKNOWLEDGMENTS}

This work is supported by the Agence Nationale de la Recherche (Contract COLORI No. ANR-12-BS04-0020).
[1] A. Schirotzek, C.-H. Wu, A. Sommer, and M. W. Zwierlein, Phys. Rev. Lett. 102, 230402 (2009).

[2] J. Catani, G. Lamporesi, D. Naik, M. Gring, M. Inguscio, F. Minardi, A. Kantian, and T. Giamarchi, Phys. Rev. A 85, 023623 (2012).

[3] C. Kohstall, M. Zaccanti, M. Jag, A. Trenkwalder, P. Massignan, G. M. Bruun, F. Schreck, and R. Grimm, Nature (London) 485, 615 (2012)

[4] K.-K. Ni, S. Ospelkaus, M. H. G. de Miranda, A. Pe'er, B. Neyenhuis, J. J. Zirbel, S. Kotochigova, P. S. Julienne, D. S. Jin, and J. Ye, Science 322, 231 (2008).

[5] T. Takekoshi, L. Reichsöllner, A. Schindewolf, J. M. Hutson, C. R. Le Sueur, O. Dulieu, F. Ferlaino, R. Grimm, and H.-C. Nägerl, Phys. Rev. Lett. 113, 205301 (2014).

[6] K. Machida, T. Mizushima, and M. Ichioka, Phys. Rev. Lett. 97, 120407 (2006)

[7] G. G. Batrouni, M. H. Huntley, V. G. Rousseau, and R. T. Scalettar, Phys. Rev. Lett. 100, 116405 (2008).

[8] G. Orso, E. Burovski, and T. Jolicoeur, Phys. Rev. Lett. 104, 065301 (2010).

[9] C. Chin, R. Grimm, P. S. Julienne, and E. Tiesinga, Rev. Mod. Phys. 82, 1225 (2010).

[10] K. Goral, T. Koehler, S. A. Gardiner, E. Tiesinga, and P. S. Julienne, J. Phys. B 37, 3457 (2004).

[11] J. W. Park, C.-H. Wu, I. Santiago, T. G. Tiecke, S. Will, P. Ahmadi, and M. W. Zwierlein, Phys. Rev. A 85, 051602(R) (2012).

[12] C.-H. Wu, J. W. Park, P. Ahmadi, S. Will, and M. W. Zwierlein, Phys. Rev. Lett. 109, 085301 (2012).
[13] J. W. Park, S. A. Will, and M. W. Zwierlein, Phys. Rev. Lett. 114, 205302 (2015).

[14] T. A. Schulze, I. I. Temelkov, M. W. Gempel, T. Hartmann, H. Knöckel, S. Ospelkaus, and E. Tiemann, Phys. Rev. A 88, 023401 (2013).

[15] H. Lefebvre-Brion and R. W. Field, The Spectra and Dynamics of Diatomic Molecules (Elsevier Academic, New York, 2004).

[16] E. Tiesinga, C. J. Williams, P. S. Julienne, K. M. Jones, P. D. Lett, and W. D. Phillips, J. Res. Natl. Inst. Stand. Technol. 101, 505 (1996).

[17] E. Tiesinga, C. J. Williams, and P. S. Julienne, Phys. Rev. A 57, 4257 (1998).

[18] F. H. Mies, Phys. Rev. A 7, 957 (1973).

[19] A. Gerdes, M. Hobein, H. Knöckel, and E. Tiemann, Eur. Phys. J. D 49, 67 (2008).

[20] P. S. Julienne and J. M. Hutson, Phys. Rev. A 89, 052715 (2014).

[21] K. Jachymski and P. S. Julienne, Phys. Rev. A 88, 052701 (2013).

[22] S. Roy, M. Landini, A. Trenkwalder, G. Semeghini, G. Spagnolli, A. Simoni, M. Fattori, M. Inguscio, and G. Modugno, Phys. Rev. Lett. 111, 053202 (2013).

[23] J. W. Park, S. A. Will, and M. W. Zwierlein, New J. Phys. 17, 075016 (2015).

[24] C. Ticknor, C. A. Regal, D. S. Jin, and J. L. Bohn, Phys. Rev. A 69, 042712 (2004).

[25] As expected, the second-order spin orbit appears to be negligible in this light species [37].

[26] M. Landini, S. Roy, G. Roati, A. Simoni, M. Inguscio, G. Modugno, and M. Fattori, Phys. Rev. A 86, 033421 (2012). 
[27] G. Salomon, L. Fouché, S. Lepoutre, A. Aspect, and T. Bourdel, Phys. Rev. A 90, 033405 (2014).

[28] R. G. Newton, Phys. Rev. 114, 1611 (1959).

[29] L. M. Duan, E. Demler, and M. D. Lukin, Phys. Rev. Lett. 91, 090402 (2003).

[30] E. Altman, W. Hofstetter, E. Demler, and M. D. Lukin, New J. Phys. 5, 113 (2003).

[31] M. A. García-March, B. Juliá-Díaz, G. E. Astrakharchik, T. Busch, J. Boronat, and A. Polls, New J. Phys. 16, 103004 (2014).

[32] T. Laue, E. Tiesinga, C. Samuelis, H. Knöckel, and E. Tiemann, Phys. Rev. A 65, 023412 (2002).
[33] C. D’Errico, M. Zaccanti, M. Fattori, G. Roati, M. Inguscio, G. Modugno, and A. Simoni, New J. Phys. 9, 223 (2007).

[34] G. Roati, M. Zaccanti, C. D’Errico, J. Catani, M. Modugno, A. Simoni, M. Inguscio, and G. Modugno, Phys. Rev. Lett. 99, 010403 (2007).

[35] F. Riboli and M. Modugno, Phys. Rev. A 65, 063614 (2002).

[36] T. Kishimoto, J. Kobayashi, K. Noda, K. Aikawa, M. Ueda, and S. Inouye, Phys. Rev. A 79, 031602 (2009).

[37] F. H. Mies, C. J. Williams, P. S. Julienne, and M. Krauss, J. Res. Natl. Inst. Stand. Technol. 101, 521 (1996). 